Provided for non-commercial research and education use. Not for reproduction, distribution or commercial use.

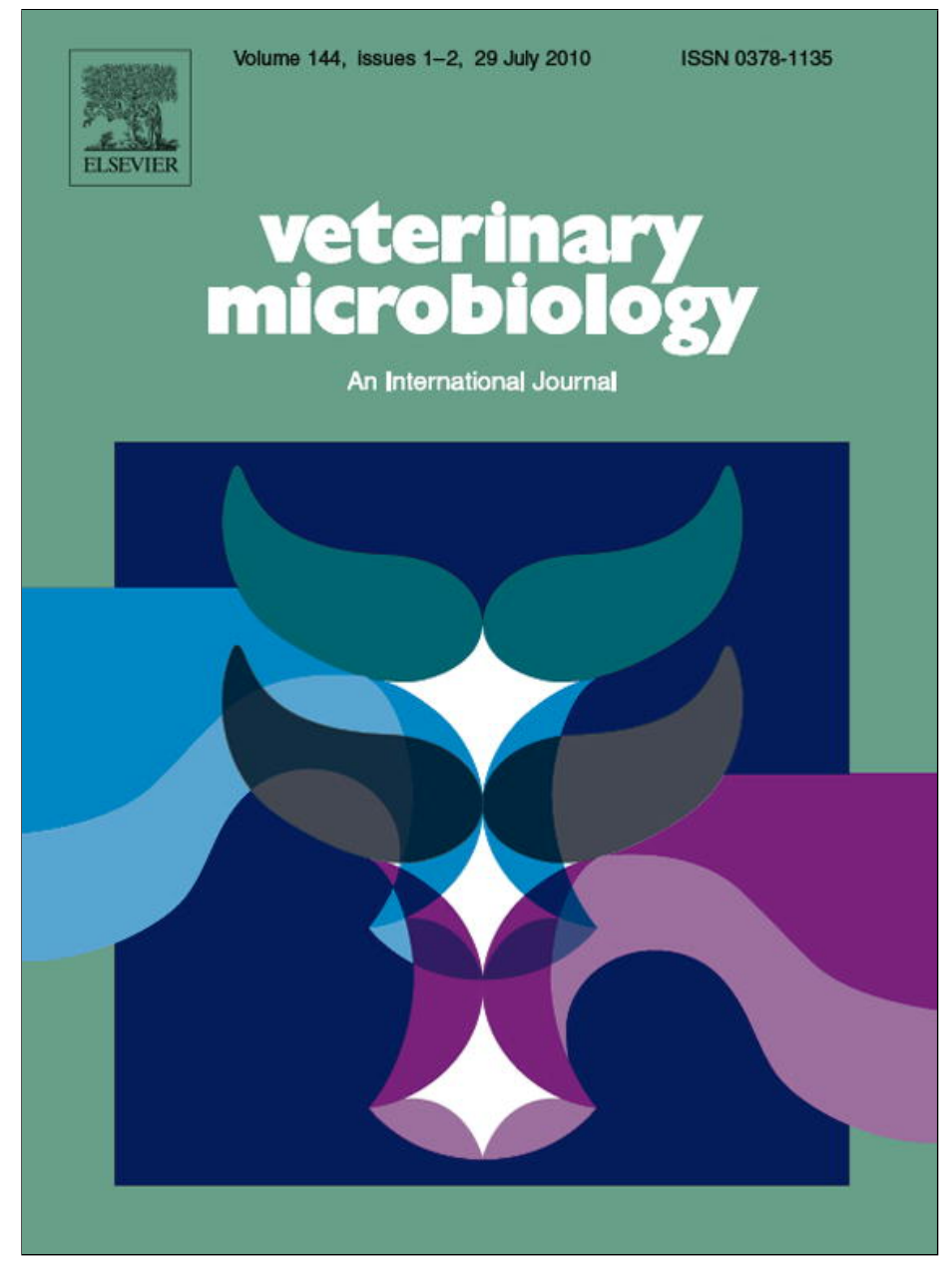

This article appeared in a journal published by Elsevier. The attached copy is furnished to the author for internal non-commercial research and education use, including for instruction at the authors institution and sharing with colleagues.

Other uses, including reproduction and distribution, or selling or licensing copies, or posting to personal, institutional or third party websites are prohibited.

In most cases authors are permitted to post their version of the article (e.g. in Word or Tex form) to their personal website or institutional repository. Authors requiring further information regarding Elsevier's archiving and manuscript policies are encouraged to visit:

http://www.elsevier.com/copyright 


\title{
Garlic derivatives (PTS and PTS-O) differently affect the ecology of swine faecal microbiota in vitro
}

\author{
Raquel Ruiz ${ }^{\text {a }}$ M.P. García ${ }^{\text {b,1 }}$, A. Lara ${ }^{\text {b }}$ L.A. Rubio ${ }^{\mathrm{a}, *}$ \\ a Dpto. de Fisiología y Bioquímica de la Nutrición Animal (IFNA, EEZ, CSIC), Profesor Albareda, 1, 18008 Granada, Spain \\ ${ }^{\mathrm{b}}$ Dpto. I+D/Area Biotecnología, DMC Research Center SL, Camino de Jayena s/n, 18620 Alhendín, Granada, Spain
}

\section{A R T I C L E I N F O}

\section{Article history:}

Received 16 September 2009

Received in revised form 14 December 2009 Accepted 18 December 2009

\section{Keywords:}

Antimicrobial

Garlic

Microbiota

Pig

\begin{abstract}
A B S T R A C T
A number of in vitro experiments were designed to evaluate the effects of two different industrial products, namely PROALLIUM-S-DMC and PROALLIUM-SO-DMC (DMC Research Center, Granada, Spain), obtained from garlic (Allium sativum) on the faecal microbiota of pigs. The effects of three different concentrations (50,200 and $400 \mathrm{ppm}$ ) of the active compounds (PTS and PTS-O, respectively) from both industrial products on the gastrointestinal microbiota of pigs were tested. Growth medium without any additive $(0 \mathrm{ppm})$ was used as control. Predominant bacterial groups (total aerobes, total anaerobes, lactobacilli, bifidobacteria, coliforms, enterobacteria, bacteroides and clostridia) were studied. Results showed that both PTS and PTS-O have significant $(P<0.01)$ antimicrobial activity against every group studied, although enterobacteria and coliforms were the most affected populations $(P<0.01)$. Time kill curves for Escherichia coli and Salmonella typhimurium, two common pathogens of pigs, showed that both compounds had a bactericidal effect against these strains. For the bacterial groups here studied, the antimicrobial effect of PTS-O was significantly $(P<0.001)$ stronger than that of PTS. Trials in vivo are in course to study the potential use of these products as alternatives to antibiotics in pig feeds.
\end{abstract}

(c) 2009 Elsevier B.V. All rights reserved.

\section{Introduction}

Both therapeutic and growth-promoter antibiotics (AGP) have been regularly used in order to improve animal performance and to prevent gastrointestinal disorders. A number of different mechanisms have been proposed on the mode of action of AGP: (1) inhibition of sub-clinical infections, (2) reduction of growth-depressing microbial metabolites, (3) reduction of microbial use of nutrients and (4) enhanced uptake and use of nutrients

Abbreviations: AGP, antibiotic growth-promoters; CFU, colony forming units; PTS, propyl propane thiosulfinate; PTS-O, propyl propane thiosulfonate.

* Corresponding author. Tel.: +34 1 958572757; fax: +34 1958572753.

E-mail addresses: luis.rubio@eez.csic.es, 1rubio@eez.csic.es (L.A. Rubio).

${ }^{1}$ Deceased (11 December 2009). through the thinner intestinal wall associated with antibiotic-fed animals (Visek, 1978; Anderson et al., 1999; Dibner and Richards, 2005). However, evidence shows that antibiotic resistance genes can be transmitted from animal to human microbiota (Greko, 2001), and the detection of pathogenic bacteria resistant to a number of antimicrobial agents (Aarestrup, 2003) have resulted in the full ban of these additives in animal nutrition in the European Union from January 2006 onwards (Anderson et al., 1999; Dibner and Richards, 2005). This new situation has promoted the investigation on alternative new feed additives with the benefits of AGP but without their observed drawbacks.

Some plant extracts have been proposed as potential alternative candidates to the use of AGP. Among those, garlic (Allium sativum) has been traditionally used for its antimicrobial, antifungal and antioxidant properties, which have been known for centuries. Recently, some 
amounts of faeces from the three animals were thawed at room temperature and immediately diluted $1: 10$ in $150 \mathrm{mM} \mathrm{NaHCO}{ }_{3}$ buffer adjusted to $\mathrm{pH} 7.4$ ( $1 \mathrm{~g}$ of faeces in $9 \mathrm{~mL}$ of buffer), and mixed in a stomacher for $2 \mathrm{~min}$. Blended, diluted faeces were filtered through miracloth (Calbiochem, 475855) and sealed in serum bottles under a $\mathrm{N}_{2}$ stream.

Appropriate sample $(n=3)$ and control $(n=3)$ tubes were aseptically inoculated with diluted faeces. Four millilitres of inoculum were added to $26 \mathrm{~mL}$ of medium (see above) in each tube under $\mathrm{N}_{2}$ stream. Sealed tubes were incubated at $37{ }^{\circ} \mathrm{C}$ with periodic mixing for $24 \mathrm{~h}$. After this time, tubes were placed into $\mathrm{a}-80^{\circ} \mathrm{C}$ freezer until used.

\subsection{Microbial analyses}

Fermentation samples were thawed at room temperature and immediately serially diluted with sterile buffered peptone water (BPW) (Cultimed, 413795) and plated in triplicate to be used in colony-counting assays. The BPW dilutions were used for counts of bacteroides, clostridia, bifidobacteria, lactobacilli, coliforms, enterobacteria, total aerobes and total anaerobes, by using the following media and procedures:

Clostridia: Reinforced clostridial agar (Oxoid, CM0151B) with $20 \mathrm{mg} / \mathrm{mL}$ of polymixin B (Sigma, P4932) after incubation at $37^{\circ} \mathrm{C}$ for $48 \mathrm{~h}$.

Bacteroides: Bile aesculin agar (Oxoid, CM0888) after incubation at $37^{\circ} \mathrm{C}$ for $24 \mathrm{~h}$.

Lactobacilli and Bifidobacteria: Man, Rogosa and Sharpe (MRS) agar (Oxoid, CM 0361) for lactobacilli and the same media supplemented with $0.5 \mathrm{mg} / \mathrm{L}$ dicloxacillin sodium salt hydrate (Sigma, D9016), $1 \mathrm{~g} / \mathrm{L} \mathrm{LiCl}$ (Sigma, L9650) and $0.5 \mathrm{~g} / \mathrm{L}$ L-cysteine hydrochloride (Sigma, C1276) for bifidobacteria. Incubations were performed at $37{ }^{\circ} \mathrm{C}$ for $48 \mathrm{~h}$ under anaerobic conditions.

Enterobacteria and Coliforms: $3 \mathrm{M}$ Petrifilm Enterobacteriaceae Count (EB) plates and $3 \mathrm{M}$ Petrifilm Coliform Count (CC) plates, respectively. Incubations were performed at $37^{\circ} \mathrm{C}$ for $24 \mathrm{~h}$.

Total aerobes: $3 \mathrm{M}$ Petrifilm Aerobic Count (AC) plates after incubation at $37^{\circ} \mathrm{C}$ for $24 \mathrm{~h}$.

Total anaerobes: Brain Heart Infusion agar (Oxoid, $\mathrm{CM} 0375$ ) after incubation at $37^{\circ} \mathrm{C}$ for $72 \mathrm{~h}$.

2.4. Bacterial strains

E. coli ATCC 25922 and S. typhimurium ATCC13311 were donated by DMC Research Center SL. Mixed cultures of enterobacteria and coliforms were obtained by isolation of these bacterial groups from pig faeces in $3 \mathrm{M}$ Petrifilm Enterobacteriaceae Count (EB) plates and $3 \mathrm{M}$ Petrifilm Coliform Count (CC) plates, respectively.

\subsection{Minimal inhibitory (MIC) and minimal bactericidal concentration $(\mathrm{MBC})$ determinations}

MIC and MBC were performed as described by Bakri and Douglas (2005). PTS and PTS-O were serially diluted in sterile LB broth in microtitre wells and each inoculated with $10 \mu \mathrm{L}$ of standardised cell suspension $\left(2 \times 10^{6} \mathrm{CFU} / \mathrm{mL}\right)$.
Wells were incubated at $37^{\circ} \mathrm{C}$ overnight and the highest dilution where no growth was observed was recorded as the MIC. For MBC testing, aliquots $(10 \mu \mathrm{L})$ of broth from wells containing no growth were plated onto LB agar and again incubated overnight at $37^{\circ} \mathrm{C}$. The highest dilution where no survivors were observed was recorded as the MBC. Controls were performed using sterile LB without PTS or PTS-O.

\subsection{Time kill curves}

Two organisms, E. coli and S. typhimurium $\left(1 \times 10^{7} \mathrm{CFU} /\right.$ $\mathrm{mL}$ ) were incubated in LB broth with PTS or PTS-O at a dilution equivalent to their MBC. Control suspensions without additives were incubated in parallel. At timed intervals, aliquots were removed, serially diluted and the number of survivors estimated on LB agar using a variation of the Miles et al. (1938) technique, which involves spotting $100 \mu \mathrm{L}$ aliquots of serial dilutions onto agar plates and counting the colonies in each inoculated spot $(n=3)$

\subsection{Statistical analysis}

Statistical analysis of data was performed by using SPSS software (SPSS 15.0 for Windows, SPSS Inc., 2006). The effects of the concentrations used [0 (control), 50, 200 and $400 \mathrm{ppm}$ ] for both active ingredients (PTS, PTS-O) on microbial counts were analyzed by the least-squares method using a model with one main effect (concentration). The effects of active ingredients (PTS, PTS-O) and concentrations (50, 200 and $400 \mathrm{ppm}$ ) on bacterial counts were analysed by the least-squares method using a model with two main effects (additive and concentration) and their interactions. An orthogonal polynomial procedure (Table 2) was used in concentrations (50, 200 and $400 \mathrm{ppm}$ ) sum of squares into its linear and quadratic components.

\section{Results}

\subsection{Effects of PTS and PTS-O on microbial populations}

Total counts of the microbial populations studied are shown in Fig. 2. The three different concentrations of PTS $(50,200$ and $400 \mathrm{ppm})$ tested resulted in a dose-dependent reduction $(P<0.01)$ of all the microbial populations studied. Enterobacteria and coliforms populations were affected the most by the presence of PTS: both populations diminished below the detection limit even in the presence of the lower concentration of PTS used in this study $(50 \mathrm{ppm})$. Bacteroides, clostridia, total aerobes and total anaerobes showed a dose-dependent reduction. Lactobacilli and bifidobacteria populations showed a similar pattern, i.e. both populations decreased in a dosedependent manner, but the effect of the addition of $400 \mathrm{ppm}$ of PTS was not different $(P=0.0634)$ from the addition of $200 \mathrm{ppm}$.

As shown in Fig. 2, the effect of PTS-O was similar to the effect of PTS: bacterial populations showed a dosedependent reduction with PTS-O addition $(P<0.01)$. The populations most affected by the presence of PTS-O were 
Table 2

Effect of the type and concentration of additive on the bacterial counts $\left[\log _{10}(\mathrm{CFU} / \mathrm{g}\right.$ faeces)].

\begin{tabular}{|c|c|c|c|c|c|c|c|c|c|}
\hline \multirow[t]{2}{*}{ Bacterial group ${ }^{1}$} & \multicolumn{2}{|l|}{ Additive } & \multicolumn{5}{|c|}{ Concentration (ppm) of active ingredient } & \multicolumn{2}{|l|}{$\mathrm{C}^{3}$} \\
\hline & PTS & PTS-O & 50 & 200 & 400 & Pooled SEM & $\mathrm{A} \times \mathrm{C}^{2}$ & $\mathrm{~L}$ & Q \\
\hline Lactobacilli & $6.558^{a}$ & $6.097^{b}$ & $8.560^{a}$ & $5.217^{b}$ & $5.206^{b}$ & 0.0369 & $*$ & $* * *$ & $* * *$ \\
\hline Bifidobacteria & $6.535^{a}$ & $6.228^{b}$ & $8.571^{a}$ & $5.273^{b}$ & $5.300^{b}$ & 0.0326 & $* * *$ & $* * *$ & $* * *$ \\
\hline Bacteroides & $7.002^{a}$ & $6.461^{b}$ & $8.898^{a}$ & $4.565^{b}$ & LOQ & 0.0259 & $* * *$ & $* * *$ & - \\
\hline Clostridia & $6.402^{\mathrm{a}}$ & $8.697^{b}$ & $8.706^{a}$ & $4.089^{b}$ & LOQ & 0.0277 & $* * *$ & $* * *$ & - \\
\hline Coliforms & $\mathrm{ND}^{\mathrm{a}}$ & $5.967^{b}$ & 5.967 & LOQ & LOQ & 0.0063 & - & - & - \\
\hline Enterobacteria & $\mathrm{ND}^{\mathrm{a}}$ & $6.108^{b}$ & 6.108 & LOQ & LOQ & 0.0230 & - & - & - \\
\hline Total aerobes & $6.529^{a}$ & $7.337^{b}$ & $8.989^{a}$ & $7.815^{b}$ & $3.995^{c}$ & 0.0748 & $* * *$ & $* * *$ & $* * *$ \\
\hline Total anaerobes & $6.650^{a}$ & $6.060^{b}$ & $8.999^{a}$ & $5.079^{b}$ & $4.987^{b}$ & 0.0491 & NS & $* * *$ & $* * *$ \\
\hline
\end{tabular}

${ }^{1}$ For each factor (additive, concentration), means in the same raw with different superscript letters differ $(P<0.01)$. NS, not significant. LOQ, too low to count.

${ }^{2} \mathrm{~A} \times \mathrm{C}$ : significance of interaction additive $\mathrm{x}$ concentration $\left({ }^{*} P<0.05 ;{ }^{* * *} P<0.001\right)$.

${ }^{3}$ Linear (L) and quadratic (Q) effects of concentrations (C). No quadratic affect could be analysed for variable A because there were only 2 additives $\left({ }^{* * *} P<0.001\right)$.

enterobacteria and coliforms, and no detectable values were recorded with the addition of over 200 ppm of PTS-O. Lactobacilli and bifidobacteria showed a similar pattern to that found with PTS. Counts of both groups slightly decreased with the addition of 50 ppm of PTS-O, while the addition of 200 and $400 \mathrm{ppm}$ of PTS-O gave place to the disappearance of one half of viable counts for both populations. Bacteroides and clostridia showed a dosedependent reduction. These populations were more sensitive to the presence of PTS-O than they were to PTS, as species of these genera could not be detected in the presence of $200 \mathrm{ppm}$ of PTS-O. Total aerobes population
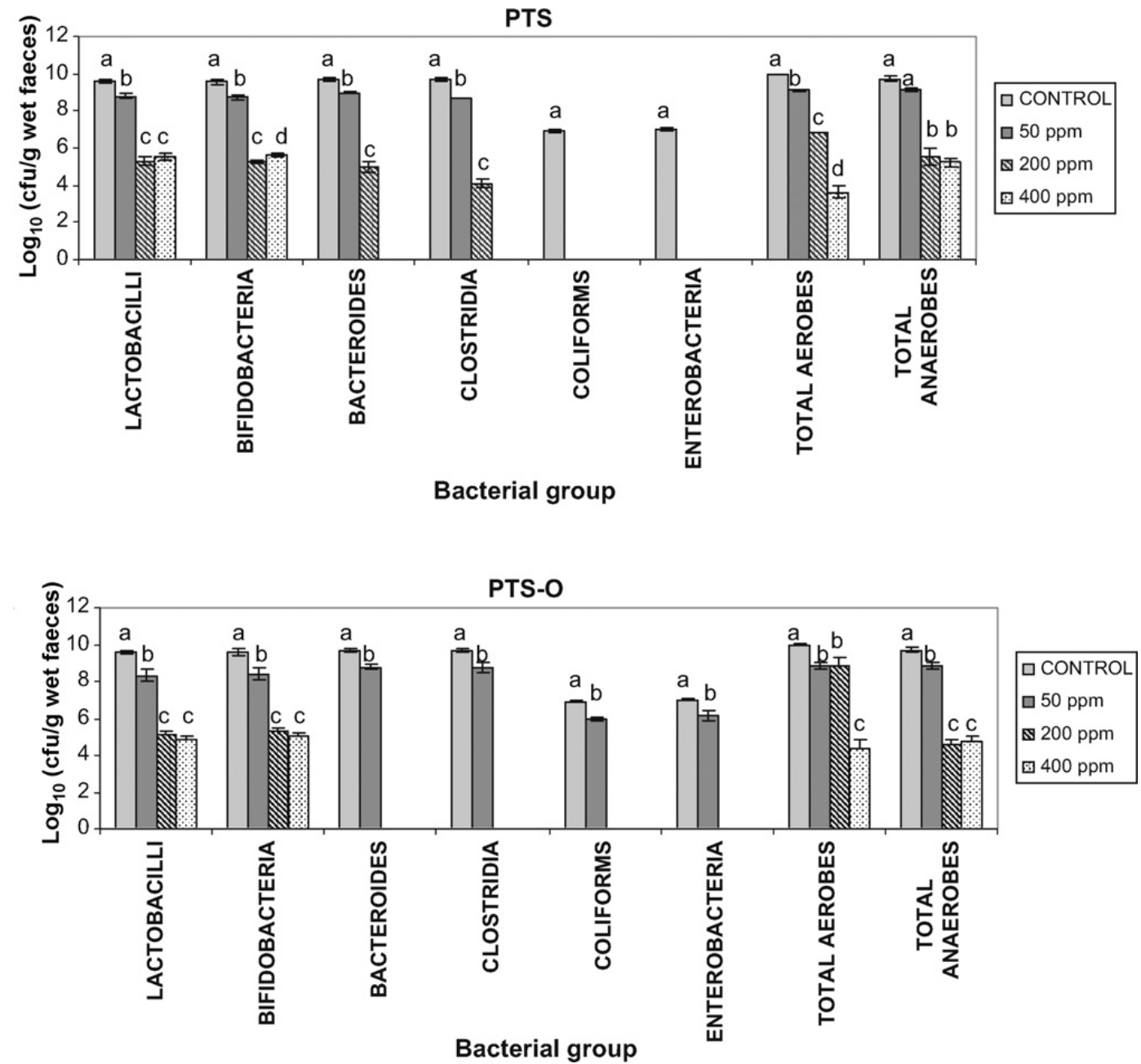

Fig. 2. Counts of lactobacilli, bifidobacteria, bacteroides, clostridia, coliforms, enterobacteria, total aerobes and total anaerobes in the presence of different amounts of (0 (control), 50, 200 and 400 ppm) of PTS or PTS-O. Mean values in each bar with different superscript letters were significantly different $(P<0.01)$ 
decreased significantly after addition of 50 and $200 \mathrm{ppm}$ of PTS-O, but the presence of $400 \mathrm{ppm}$ of the additive caused the loss of more than a half of the group. Total anaerobes were more sensitive than total aerobes to the presence of PTS-O, this population showing a significant decrease in the presence of 200 ppm of PTS-O.

Two-way ANOVA analysis (Table 2) showed that PTS-O had stronger $(P<0.001)$ antimicrobial activity than PTS for lactobacilli, bifidobacteria, bacteroides, clostridia and total anaerobes populations, while PTS was clearly more active $(P<0.001)$ than PTS-O against enterobacteria, coliforms and total aerobes. The addition of 200 and $400 \mathrm{ppm}$ of PTS and PTS-O caused similar effects in lactobacilli, bifidobacteria, coliforms, enterobacteria and total anaerobes populations. Bacteroides, clostridia and total aerobes showed a dose-dependent decrease in the presence of both compounds. Coliforms and enterobacteria were the groups most affected by the addition of PTS or PTS-O. A very significant decrease $(P<0.001)$ in viable counts was found with the addition of $50 \mathrm{ppm}$ of both additives in the media. Doses of $200 \mathrm{ppm}$ or higher gave place to the disappearance of any viable cell of these genera in the conditions here used.

\section{(A) Escherichia coli}

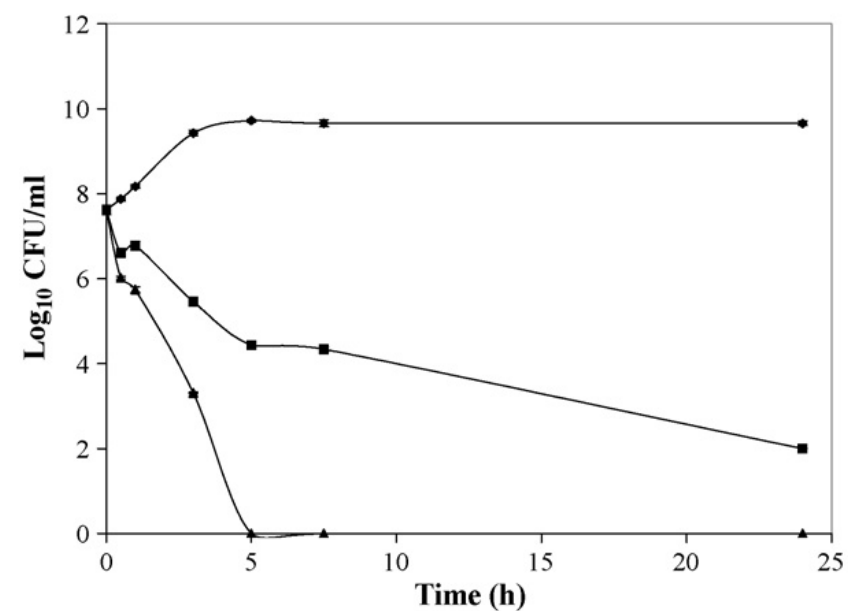

(B) Salmonella Typhimurium

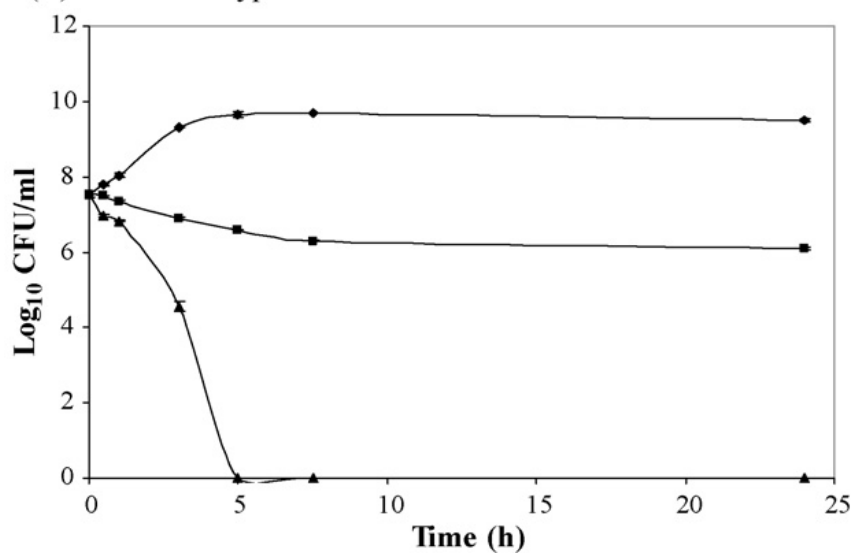

Fig. 3. Time kill curve results for E. coli (Panel A) and S. typhimurium (Panel B) tested against $100 \mathrm{ppm}$ of PTS $(\boldsymbol{\square})$ or PTS-O ( $\mathbf{\Delta})$. Control cells suspensions without additives $(\bullet)$ were incubated in parallel. Data are expressed as $\log _{10} \mathrm{CFU} / \mathrm{mL}$ recovered on $\mathrm{LB}$ agar at each time point. Values are means with SD in bars.

\subsection{Time kill curves in the presence of PTS and PTS-O}

Time kill curves were performed for two common enteric pig pathogens, namely E. coli and S. typhimurium (Fig. 3). Both species showed identical MIC (100 ppm) and MBC $(100 \mathrm{pm})$ values for PTS and PTS-O. For both E. coli and $S$. typhimurium, PTS-O showed a higher $(P<0.01)$ activity than PTS. Killing of $E$. coli and $S$. typhimurium began almost immediately after incubation with PTS-O. After $5 \mathrm{~h}$ of incubation, no viable cells were recovered. E. coli was more sensitive $(P<0.01)$ than $S$. typhimurium to the presence of PTS, showing a drop greater than 5 log units in viability after $24 \mathrm{~h}$ of incubation. Control cultures without additives showed an exponential plus a stationary growth phase, with no drop in viability over the same period.

Time kill curves were also performed for mixed cultures of enterobacteria and coliforms isolated from pig faeces. Results (Fig. 4) showed that for both groups PTS-O showed a higher $(P<0.01)$ activity than PTS. Coliforms were more sensitive $(P<0.01)$ than enterobacteria to the action of both compounds. These results are in agreement with those obtained with pure cultures of $E$. coli and $S$. typhimurium.

(A) Enterobacteria

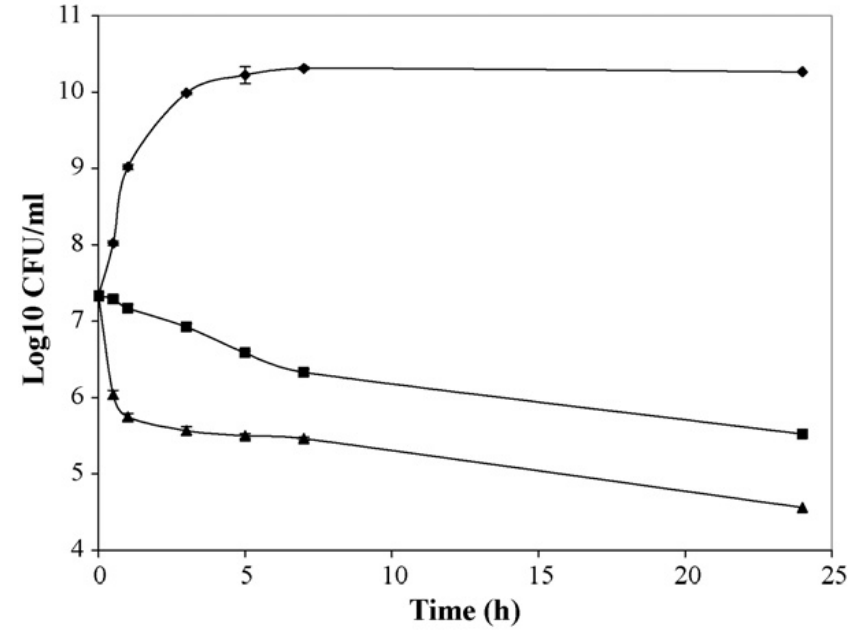

(B) Coliforms

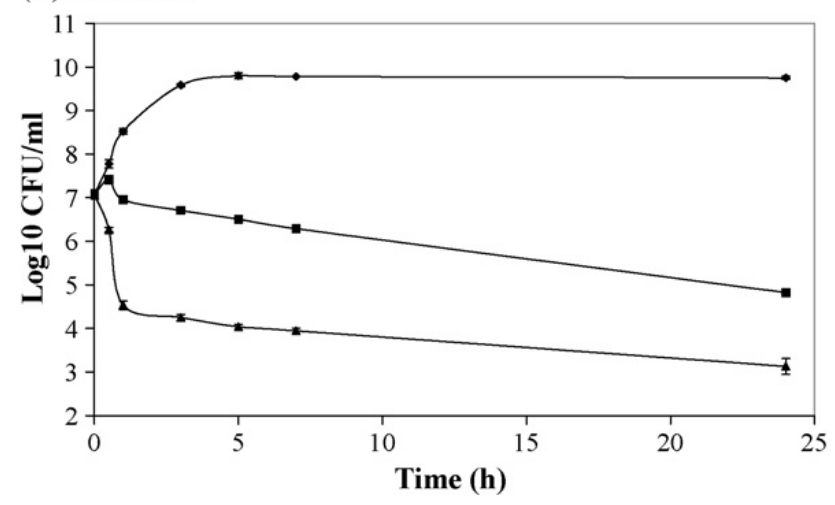

Fig. 4. Time kill curve results for enterobacteria (Panel A) and coliforms (Panel B) tested against 100 ppm of PTS $(\boldsymbol{\square})$ or PTS-O $(\boldsymbol{\Delta})$. Control cells suspensions without additives $(\bullet)$ were incubated in parallel. Data are expressed as $\log _{10} \mathrm{CFU} / \mathrm{mL}$ recovered on LB agar at each time point. Values are means with SD in bars. 
Cultures containing only the inert commercial alimentary support (where PTS and PTS-O were incorporated to produce the commercial preparations) as additive showed a behaviour similar to that of cultures without additives (data not shown).

\section{Discussion}

Among the biologically active sulfur species in garlic, allicin has played the major role in garlic research. Allicin (Fig. 1a) is formed from the chemically rather unreactive sulfoxide precursor alliin (Fig. 1b) in a reaction catalysed by the C-S-lyase enzyme alliinase. Chemically speaking, allicin is a thiosulfinate, a reactive sulfur species which kills various bacteria, fungi, yeasts and even cancer cells. Decomposition and degradation of allicin results in a range of "second generation" compounds (diallylsufide, diallyldisulfide, diallyltrisulfide and diallyltetrasulfide) frequently found in garlic extracts, such as garlic oils. Other decomposition products of allicin include the dithiins 3-vinyl-3,4-dihydro-1,2-dithiin and 2-vinyl-2,4dihydro-1,3-dithiin and more complex chemical structures, such as E-ajoene and $Z$-ajoene (Fig. 1e). Follow-on reactions of allicin and polysulfides with intracellular thiols result in additional sulfur species, such as $S$ allylmercaptocystein. Each of these sulfur species exhibits its own chemical properties and biochemical activity. The central role of allicin in garlic chemistry is presently being challenged by a number of findings which have confirmed antibiotic and anticancer activities for diallylsulfides similar or even superior to the ones of allicin (Münchberg et al., 2007).

The interest on the beneficial properties of products derived from garlic has risen in the last decades due to its cardiovascular, antineoplastic and antimicrobial properties (Harris et al., 2001; Ariga and Seki, 2006). In particular, a wide range of microorganisms including Gram-positive and Gram-negative pathogenic bacteria, fungi, protozoa and viruses have been shown to be sensitive to crushed garlic preparations (Ankri and Mirelman, 1999; Harris et al., 2001; Ruddock et al., 2005). Antibacterial properties of garlic against some enteric bacteria have been previously described by Ross et al. (2001). These authors demonstrated that products such as garlic oil and garlic powder inhibited the growth of a range of Gram-positive and Gram-negative enteric bacteria, including both pathogenic and commensal bacteria.

Results showed that both PTS and PTS-O had a significant $(P<0.05)$ antimicrobial effect in vitro against all populations of intestinal microbiota tested. The antibacterial activity of garlic is widely attributed to allicin, the major thiosulfinate in garlic (Cavallito and Bailey, 1944). Thiosulfinates (as allicin and PTS) are organosulfur compounds originated by the transformation of $S$-alk(en)yl-L-cysteine sulfoxides (ACSOs) by the enzyme alliinase. ACSOs are cleaved by alliinase to sulfenic acids, ammonia, and pyruvate. Sulfenic acids are highly reactive and transient compounds. In fresh tissue, sulfenic acids with the same or different alk(en)yl substituents condense to form thiosulfinates. Thiosulfinates have been implicated as a principle source of the antiplatelet property of raw onion and garlic juice. In fact, allicin and PTS have a significantly greater antiplatelet potency than aspirin (Briggs et al., 2000).

More importantly in the present context, thiosulfinates derived from garlic have also shown antimicrobial activity against Gram-positive and Gram-negative bacteria (Yoshida et al., 1999). The main antimicrobial effect of thiosulfinates as allicin has been reported to be due to its chemical reaction with thiol groups of various enzymes such as the acetyl-CoA-forming system, consisting of acetate kinase and phosphotransacetyl-CoA synthetase (Focke et al., 1990). RNA polymerase might also be a target for allicin. In $S$. typhimurium, allicin at bacteriostatic concentrations inhibits RNA synthesis (Feldberg et al., 1988). This same enzyme contains in E. coli a single sulfhydryl group which might react with allicin (Ozolin et al., 1990). The significance of allicin as a biological effector molecule is not only due to its high reactivity with low and high molecular weight thiols, but also to its accessibility resulting from high membrane permeability (Miron et al., 2000). The condensation product of allicin, ajoene (Fig. 1e), which has a similar oxygenated sulfur group also showed a broad-spectrum antimicrobial activity, inhibiting the growth of Gram-positive and Gram-negative bacteria. In this case, the disulfide bond in ajoene appears to be necessary for its antimicrobial activity (Naganawa et al., 1996).

Thiosulfonates (such as PTS-O) have only sporadically been studied to date. Oxidation of a disulfide or thiosulfinate is possible thiosulfonate formation pathways, although the reaction of certain C-S-lyase cleavage products might also result in this chemotype. Like thiosulfinates, thiosulfonates react readily with thiols, yet are less potent as oxidants. The antimicrobial activity of some thiosulfonates has also been previously reported. For example, pseudoallicin, a thiosulfonate found in garlic, possess about half the antibacterial activity of allicin. Thiosulfonates, which possess a similar reactivity, are more stable than thiosulfinates (Jacob, 2006).

Although the two compounds here assayed were active against all bacterial strains tested, either pathogenic or not, their activity was not the same. While PTS was more active against coliforms and enterobacteria, PTS-O showed higher activity against lactobacilli, bifidobacteria, bacteroides and clostridia (Fig. 2). The groups most sensitive to the action of PTS and PTS-O were enterobacteria and coliforms, which were not detectable in the presence of the lower concentration of PTS here used (50 ppm). On the contrary, lactobacilli and bifidobacteria were the most resistant populations to the action of PTS and PTS-O. These results are in agreement with the antibacterial effects of garlic previously described in the literature. Thus, it has been shown that garlic products exert a broad-range of antimicrobial activity against Grampositive and Gram-negative bacteria, including species of Escherichia, Salmonella, Klebsiella, Streptococcus, Lactobacillus, Bacillus, Bacteroides, Proteus (Ross et al., 2001), Staphylococcus, Micrococcus, and Clostridium (Sharma et al., 1977; De Witt et al., 1979; Ruddock et al., 2005). Even acid-fast bacteria such as Mycobacterium are sensitive to garlic (Uchida et al., 1975; Delaha and Garagusi, 1985). Garlic extracts are also effective against Helicobacter pylori, the 
causal agent of gastric ulcers (O'Gara et al., 2000). Also, Rees et al. (1993) documented that garlic exerts differential inhibition between beneficial intestinal microflora and potentially harmful enterobacteria, and lactic acid bacteria were the least sensitive microorganisms to the inhibitory effects of garlic. In mixed culture studies of Lactobacillus acidophilus and E. coli, garlic prevented the establishment of the last one.

A differential effect of PTS and PTS-O was also reflected in the time kill curves performed in this study for $E$. coli and S. typhimurium, two of the most important pathogens of pigs responsible for great economic losses due to mortality, morbidity, decreased growing rate, cost of medication, etc. (Moxley and Duhamel, 1999; Fairbrother et al., 2005). Killing of both strains was much faster in the presence of PTS-O, and PTS was more effective against $E$. coli than it was against $S$. typhimurium. Apart from reactivity and reaction stoichiometry, these differences are likely to be related with differences in the structure (Fig. 1c and d) or chemical stability of both compounds (see above). Also, the differential inhibition showed by PTS and PTS-O may be due to differing composition of bacterial membranes and their permeability to these compounds (Miron et al., 2000). Anyway, the same results were obtained when time kill curves were performed for enterobacteria and coliforms isolated from pig faeces. As the effects were not so strong as they were with identified strains, the bactericidal effect is probably not the same against all bacterial strains of these genera present in the pig intestine.

Based on the results found in the current study, further work is merited to establish the effectiveness and possible mechanism of action of both compounds in vivo. In fact, the results found in a preliminary in vivo trial carried out with a limited number of growing pigs (data not shown) were promising and comparable to those in vitro here described. Thus, the inclusion of PTS-O in an experimental pig diet free of antibiotics gave place to higher faecal counts of the lactic bacteria strains and lower of coliforms and enterobacteria than a commercial diet used as control which contained formic and propionic acids as antimicrobial agents.

\section{Conclusions}

In conclusion, the active ingredients of PROALLIUM-SDMC and PROALLIUM-SO-DMC (PTS and PTS-O, respectively), two organosulfurate compounds industrially obtained by decomposition of initial compounds present in garlic such as alliin and allicin, have antimicrobial activity against every bacterial group of pig faces studied, although enterobacteria and coliforms were the most affected populations. Furthermore, both products had a bactericidal effect against E. coli and S. typhimurium, two common pathogens of pigs.

\section{Acknowledgements}

Authors are grateful to Drs. L. Lara and E. Rubio, for excellent technical assistance. R. Ruiz acknowledges receipt of an I3P contract.

\section{References}

Aarestrup, F.M., 2003. Effects of termination of AGP use on antimicrobial resistance in food animals. Pages 6-11 in Working papers for the WHO international review panels evaluation. Document WHO/CDS/CPE/ ZFK/2003.1a. World Health Organization, Geneva, Switzerland.

Anderson, D.B., McCracken, V.J., Aminov, R.I., Simpson, J.M., Mackie, R.I., Verstegen, M.W.A., Gaskins, H.R., 1999. Gut microbiology and growth-promoting antibiotics in swine. Pig News Inf. 20 (4), $115 \mathrm{~N}-122 \mathrm{~N}$.

Ankri, S., Mirelman, D., 1999. Antimicrobial properties of allicin from garlic. Microbes Infect. 2, 125-129.

Ariga, T., Seki, T., 2006. Antithrombotic and anticancer effects of garlicderived sulphur compounds: a review. Biofactors 26 (2), 93-103.

Bakri, I.M., Douglas, C.W.I., 2005. Inhibitory effect of garlic extract on oral bacteria. Arch. Oral Biol. 50, 645-651.

Briggs, W.H., Xiao, H., Parkin, K.L., Shen, C., Goldman, I.L., 2000. Differential inhibition of human platelet aggregation by selected Allium thiosulfinates. J. Agric. Food Chem. 48 (11), 5731-5735.

Cavallito, C., Bailey, J.H., 1944. Allicin, the antibacterial principle of Allium sativum. Isolation, physical properties and antibacterial action. J. Am. Chem. Soc. 66, 1944-1952.

De Witt, J.C., Notermans, S., Gorin, N., Kampelmacher, E.H., 1979. Effect of garlic oil or onion oil on toxin production by Clostridium botulinum in meat slurry. J. Food Prot. 42, 222-224.

Delaha, E.C., Garagusi, V.F., 1985. Inhibition of mycobacteria by garlic extract (Allium sativum). Antimicrob. Agents Chemother. 27, 485486.

Dibner, J.J., Richards, J.D., 2005. Antibiotic growth promoters in agriculture: history and mode of action. Poult. Sci. 84, 634-643.

Fairbrother, J.M., Nadeau, E., Gyles, C.L., 2005. Escherichia coli in postweaning diarrhea in pigs: an update on bacterial types, pathogenesis, and prevention strategies. Anim. Health Res. Rev. 6 (1), 17-39.

Feldberg, R.S., Chang, S.C., Kotik, A.N., Nadler, M., Neurwirth, Z., Sundstrom, D.C., Thompsom, N.H., 1988. In vitro mechanism of inhibition of bacterial cell growth by allicin. Antimicrob. Agents Chemother. 32, 1763-1768.

Focke, M., Feld, A., Lichtenthaler, K., 1990. Allicin, a naturally occurring antibiotic from garlic, specifically inhibits acetyl-CoA synthetase. FEBS Lett. 261, 106-108.

Greko, C., 2001. Safety aspects on non-use of antimicrobials as growth promoters. In: Piva, A., Bach Knudsen, K.E., Lindberg, J.E. (Eds.), Gut Environment of Pigs. Nottingham University Press, Nottingham, UK, pp. 219-230.

Harris, J.C., Cottrell, S.L., Plummer, S., Lloyd, D., 2001. Antimicrobial properties of Allium sativum (garlic). Appl. Microbiol. Biotechnol. 57 (3), 282-286.

Iberl, B., Winkler, G., Muller, B., Knobloch, K., 1990. Quantitative determination of allicin and alliin from garlic by HPLC. Planta Med. 56 (3), 320-326.

Jacob, C., 2006. A scent of therapy: pharmacological implications of natural products containing redox-active sulfur atoms. Nat. Prod. Rep. 23, 851-863.

Miles, A.A., Misra, S.S.K., Irwin, J.O., 1938. The estimation of the bactericidal power of the blood. J. Hygiene 38, 732-749.

Miron, T., Rabinkov, A., Mirelman, D., Wilchek, H., Weiner, L., 2000. The mode of action of allicin: its ready permeability through phospholipid membranes may contribute to its biological activity. Biochim. Biophys. Acta $1463,20-30$

Moxley, R.A., Duhamel, G.E., 1999. Comparative pathology of bacterial enteric diseases of swine. Adv. Exp. Med. Biol. 473, 83-101.

Münchberg, U., Anwar, A., Mecklenburg, S., Jacob, C., 2007. Polysulfides as biologically active ingredients of garlic. Org. Biomol. Chem. 5 (10), 1505-1518.

Naganawa, R., Iwara, N., Ishikawa, K., Fukuda, H., Fujino, T., Suzuki, A., 1996. Inhibition of microbial growth by ajoene, a sulphur-containing compound derived from garlic. Appl. Environ. Microbiol. 62 (11), 4238-4242.

O'Gara, E.A., Hill, D.J., Maslin, D.J., 2000. Activities of garlic oil, garlic powder and their diallyl constituents against Helicobacter pylori. Appl. Environ. Microbiol. 66, 2269-2273.

Ozolin, O.N., Uteshev, T.A., Kim, I.A., Deev, A.A., Kamzolova, S.G., 1990. Specific modification of the alpha-subunit of Escherichia coli RNAS polymerase by monomercuric derivative of fluorescein mercuric acetate. Mol. Biol. (Mosk.) 24, 1057-1066.

Rees, L.P., Minney, S.F., Plummer, N.T., Slater, J.H., Skyrme, D.A., 1993. A quantitative assessment of the anti-microbial activity of garlic (Allium sativum). World J. Microbiol. Biotechnol. 9, 303-307.

Ross, Z.M., O’Gara, E.A., Hill, D.J., Sleightholme, H.V., Maslin, D.J., 2001. Antimicrobial properties of garlic oil against human enteric bacteria: 
evaluation of methodologies and comparisons with garlic oil sulfides and garlic powder. Appl. Environ. Microbiol. 67 (1), 475-480.

Ruddock, P.S., Liao, M., Foster, B.C., Lawson, L., Arnason, J.T., Dillon, J.A

2005. Garlic natural health products exhibit variable constituent levels and antimicrobial activity against Neisseria gonorrhoeae, Staphylococcus aureus and Enterococcus faecalis. Phytother. Res. 19 (4), 327-334.

Sharma, V.D., Sethi, M.S., Kumar, A., Rarotra, J.R., 1977. Antibacterial property of Allium sativum Linn. In vivo and in vitro studies. Indian J. Exp. Biol. 15, 466-468.

Smiricky-Tjardes, M.R., Grieshop, C.M., Flickinger, E.A., Bauer, L.L., Fahey Jr., G.C., 2003. Dietary galactooligosaccharides affect ileal and total- tract nutrient digestibility, ileal and fecal bacterial concentrations, and ileal fermentative characteristics of growing pigs. J. Anim. Sci. 81, 2535-2545.

Uchida, Y., Takahashi, T., Sato, N., 1975. The characteristics of the antibacterial activity of garlic. Jpn. J. Antibiot. 28, 638-642.

Visek, W.J., 1978. The mode of growth promotion by antibiotics. J. Anim. Sci. 46, 1447-1469.

Yoshida, H., Katsuzaki, H., Ohta, R., Ishikawa, K., Fukuda, H., Fujino, T., Suzuki, A., 1999. Antimicrobial activity of the thiosulfinates isolated from oil-macerated garlic extract. Biosci. Biotechnol. Biochem. 63 (3), 591-594. 